\title{
DESIGN AND ANALYSIS OF DIFFERENT TECHNIQUES FOR ANALOG-TO-DIGITAL CONVERSION OF VIBRATION SIGNALS FOR WIRELESS MEASUREMENT SYSTEMS*
}

UDC ((621.317:004.421)+(681.335.2:621.395.38))

\section{Milan Dinčić, Dragan Denić, Zoran Perić}

University of Niš, Faculty of Electronic Engineering, Republic of Serbia

\begin{abstract}
The aim of this paper is to design, analyze and compare four different systems for ADC (analog-to-digital conversion) of vibration signals. Measurement of vibration signals is of particular importance in many areas, such as predictive maintenance or structural health monitoring. Wireless systems for vibration measurements become very topical, due to much easier and cheaper installation compared to wired systems. Due to the lack of transmission bandwidth and energy in wireless measurement systems, the amount of digital data being sent has to be reduced; hence, we have to apply ADC systems that can achieve the required digital signal quality, reducing the bit-rate. Four $A D C$ systems are analyzed, for possible application in wireless measurement systems: PCM (pulse code modulation) based on uniform quantization; DPCM (differential PCM) to exploit high correlation of vibration signals; two adaptive ADC systems to cope with significant variations of characteristics of vibration signals in time - APCM (adaptive $P C M$ ) with adaptation on variance and ADPCM (adaptive DPCM), with double adaptation (both on variance and correlation). These ADC models are designed and optimized specifically for vibration signals, based on the analysis of 20 vibration signals from a referent database. An experiment is done, applying designed ADC systems for digitalization of vibration signals. APCM, DPCM and ADPCM systems allow a significant bit-rate reduction compared to the PCM system, but with the increasing of complexity, hence the compromise between the bit-rate reduction and complexity is needed.
\end{abstract}

Key words: vibration measurements, wireless measurement systems, analog-to-digital conversion, adaptive quantization, Gaussian distribution

Received July 03, 2018

Corresponding author: Milan R. Dinčić

Faculty of Electrical Engineering, Aleksandra Medvedeva 14, 18000 Niš, Republic of Serbia E-mail: milan.dincic@elfak.ni.ac.rs

* Acknowledgement: This paper was realized as a part of the projects TR-32045 and III-42009, financed by the Ministry of Education and Science of the Republic of Serbia. 


\section{INTRODUCTION}

Measurement systems for vibration measurement have significant applications in many different areas (e.g. for predictive maintenance of machines in industry, where machine vibrations are used for early detection of defects and malfunctions; for structural health monitoring of large infrastructure objects (bridges, dams, large buildings, etc.) where vibrations are used for early detection of hidden cracks) [1-4]. In the recent years, wireless measurement systems (e.g. wireless sensor networks) have a particularly important role in vibration measurements, due to the numerous advantages they have in relation to wired measurement systems [5-7]. First of all, wireless measurement systems are much cheaper and easier to install. Also, wireless measurement systems can be installed in places where it is not possible to install wired measurement systems (e.g. on rotary machines). The problem of wireless measurement systems is the lack of transmission bandwidth, which makes it very difficult to achieve high bit rates. Therefore, it is desirable to digitize the vibration signals with as few bits as possible. Also, most commonly, wireless measurement systems are battery-powered, so the lack of energy is one of the most important problems, whereby most of the energy is being spent on data transmission. Hence, in order to save energy, there is a need to reduce the amount of digital data being sent by reducing the number of bits used for digital representation of samples. We can conclude that it is necessary (due to the lack of bandwidth and energy) to design an analog-to-digital conversion system that can achieve the required digital signal quality with as few bits per sample as possible (i.e. with the lowest possible bit rate).

In order to achieve optimal performances, statistical characteristics of the signal (primarily the statistical distribution of the signal amplitude) should be taken into account during the design of analog-to-digital conversion (ADC) systems. It is known that a lot of measurement signals can be modeled very well by the Gaussian distribution [8], hence it will be used in this paper.

The variance (power) of vibration signals may vary in a very wide range, while an ADC system is always designed for some specific referent variance [8]. Therefore, performances of ADC systems depend on the signal variance: the best performances are achieved when the variance of the vibration signal is the same (or very close) to the referent variance; on the other hand, by distancing the signal variance from the referent variance, a significant decrease in performances occurs. Hence, performances of ADC systems should be analyzed in the wide variance range and ADC systems should be designed in the way to achieve a satisfactory quality of the digitized signal in the whole variance range of interest.

The aim of this paper is to design, analyze and compare four different systems for analog-to-digital conversion of vibration signals. The first ADC system is the PCM (pulse code modulation) system based on uniform quantization [8], which is mostly used for its simplicity. The disadvantage of this system is that it is not robust, i.e. the SNR (signalto-noise ratio) drops significantly as the signal variance is moving away from the referent variance. Therefore, in order to achieve the desired performances in the wide variance range, we have to use a high-resolution PCM system, using a large number of bits for digitalization of vibration signals. The second ADC system is the APCM (adaptive PCM) system $[9,10]$, which is adaptable on the signal variance, giving almost constant SNR in the very wide variance range. In this way, the desired performances can be achieved with smaller number of bits. Since the vibration signals are usually highly correlated [11], with 
very close values of consecutive samples and very small values of their differences, the third ADC system is the DPCM (differential PCM) system based on quantization and coding of differences of consecutive samples, in order to exploit high signal correlation. A DPCM system can achieve the desired SNR level with a smaller number of bits compared to a PCM system. Since the variance of differences of consecutive samples, as well as the value of signal correlation, can significantly vary in time, the fourth ADC system is the ADPCM (adaptive DPCM) system [8], where double adaptation (both on the variance and on the correlation) is performed, further reducing the required number of bits in relation to a DPCM system.

These ADC systems have been analyzed in literature [8-10] for some other types of signals. This paper is devoted to design and analysis of these ADC systems specifically for vibration signals, taking into account their characteristics. Because of that, the analysis of 20 vibration signals taken from the referent RealVibration signal database [12] is performed, calculating their charactristics (the variance range, the range of the correlation coefficient, the variance range of differences of adjacent signal samples). The obtained results are used for the design and calculation of parameters of four ADC systems, with the aim to achieve required minimal SNR value for all considered vibration signals.

Experiments are done, by applying designed four ADC systems for the analog-to-digital conversion of the considered 20 vibration signals. On the basis of the experimental results, we can conclude that if we want to achieve the minimal acceptable SNR value of $23 \mathrm{~dB}$ for all considered vibration signals, we can do this with the bit rate of 8 bps (bits per sample) using the PCM system, with the bit rate of 5 bps using the APCM system, with the bit rate of 4 bps using the DPCM system and with the bit rate of 2 bps using the ADPCM system. Of course, one should keep in mind that the PCM system is the simplest of all four considered systems; APCM and DPCM are more complex while ADPCM is the most complex, so a trade-off between performances and complexity has to be made. This paper describes in details the structure, design and performances of those four ADC systems, giving the ability to each user to select the best system for its application, taking into account both performances and complexity.

The paper is organized in the following way. The second section presents the theoretical bases of all four ADC systems considered in this paper. In the first part of the third section, a detailed analysis of vibration signals from RealVibration database is performed and the basic characteristics (the variance range, the range of the correlation coefficient, etc.) of each signal are shown. On the basis of this analysis, four ADC systems are designed and experimental results (obtained by applying those four ADC systems on 20 vibration signals) are presented in the second part of the third section. Conclusion and the list of references are given at the end of the paper.

\section{THEORETICAL BACKGROUND}

In this section we will provide some theoretical background about the four ADC systems that will be considered in this paper.

\subsection{PCM system based on the uniform quantization}

PCM (pulse code modulation) is the basic system for digitalization of analog signals, where samples of the analog signal are quantized and coded with the natural binary code. 
Quantization, as the main part of the PCM system, will be the main topic of our further discussion, focusing on the PCM system based on the uniform quantization.

Quantization is one of the main steps in the process of analog-to-digital conversion. By quantization, each sample of an analog signal (which can take any analog value from a certain continuous range), is mapped into one (nearest) discrete value from a set of $N$ allowed discrete values. In $N$-level quantization, the range of values of the analog signal is divided into $N$ quantization intervals. The boundaries between intervals are called thresholds, $x_{i}, i=0, \ldots, N$. Within each quantization interval there is one representation level, $y_{i}, i=0, \ldots, N$. All analog values $x$ from the $i$-th interval $\left(x_{i-1}, x_{i}\right)$ are mapped to the representation level for that interval $y_{i}$. Mathematically, quantization can be defined as mapping $Q: \mathfrak{R} \rightarrow\left\{y 1, \ldots y_{N}\right\}, Q(x)=y_{i}$, $\forall x \in\left(x_{i-1}, x_{i}\right)$, where $\mathfrak{R}$ represents the set of real numbers. The quantizer is completely defined by the set of its thresholds and representation levels.

In order to achieve optimal performances, the statistical distribution of the signal should be taken into account when the quantizer is designed. A lot of measurement signals, including vibration signals, can be very well modeled by the zero-mean Gaussian distribution, whose probability density function is defined by the following expression:

$$
p(x)=\frac{1}{\sqrt{2 \pi} \sigma} e^{-\frac{x^{2}}{2 \sigma^{2}}},
$$

where $\sigma^{2}$ denotes the variance (power) of the signal.

Quantizers are always designed for some referent variance $\sigma_{0}^{2}$ and they have the highest performances if the signal variance $\sigma^{2}$ is equal (or very close) to the referent variance $\sigma_{0}^{2}$. As the signal variance becomes more distant from the referent variance, quantizer performances decrease more. Signal variance can vary in a quite wide range; hence the referent variance should be carefully chosen to maximize the average performances of the quantizer.

The most commonly used type of quantizers, because of simplicity, is a uniform quantizer, where all quantization intervals have the same width $\Delta$ and the representation levels are in the middle of the quantization intervals. The range of the quantizer is $\left(-x_{\max }, x_{\max }\right)$, where $x_{\max }=x_{N}=-x_{0}$ denotes the maximal amplitude of the quantizer. For the $N$ - level uniform quantizer, the quantization stepsize is $\Delta=2 x_{\max } / N$. Thresholds of the uniform quantizer are defined as $x_{i}=-x_{\max }+i \cdot \Delta, i=0, \ldots, N$, while representation levels are defined as $y_{i}=-x_{\max }+(i-1 / 2) \cdot \Delta, i=1, \ldots, N$. Expressions for the thresholds and representation levels can be written in the following form:

$$
x_{i}=(-N / 2+i) \cdot \Delta, i=0, \ldots, N ; \quad y_{i}=(-N / 2+i-1 / 2) \cdot \Delta, i=1, \ldots, N .
$$

Since the probability density function $p(x)$ is symmetric around 0 , the uniform quantizer will be also symmetric around 0 , which means: $x_{N / 2}=0, x_{N / 2+i}=-x_{N / 2-i}, y_{N / 2+i}=-y_{N / 2-i}$, $i=1, \ldots, N / 2$. The uniform quantizer is completely determined with the stepsize $\Delta$; if we find optimal value of $\Delta$, we can calculate all the thresholds and the representational levels $x_{i}$ and $y_{i}$.

Distortion $D$ represents errors occurred during the quantization. It is equal to the sum of granular distortion $D_{g}$ and overload distortion $D_{o v}$. Granular distortion represents quantization errors for signal samples within the quantizer range $\left(-x_{\max }, x_{\max }\right)$, while 
overload distortion represents quantization errors for signal samples outside the quantizer range. Granular and overload distortions are defined with the following expressions:

$$
\begin{gathered}
D_{g}=2 \sum_{i=1}^{N / 2} \int_{x_{N / 2+i-1}}^{x_{N / 2+i}}\left(x-y_{N / 2+i}\right)^{2} p(x) d x=2 \sum_{i=1}^{N / 2} \int_{(i-1) \Delta}^{i \cdot \Delta}(x-(i-1 / 2) \cdot \Delta)^{2} \frac{1}{\sqrt{2 \pi} \sigma} e^{-\frac{x^{2}}{2 \sigma^{2}}} d x, \\
D_{o v}=2 \int_{x_{N}}^{+\infty}\left(x-y_{N}\right)^{2} p(x) d x=2 \int_{x_{\max }}^{+\infty}(x-(N-1) \cdot \Delta / 2)^{2} \frac{1}{\sqrt{2 \pi} \sigma} e^{-\frac{x^{2}}{2 \sigma^{2}}} d x .
\end{gathered}
$$

Due to the symmetry, distortions in the positive and negative parts of the quantizer are the same. Therefore, we can consider only distortion in the positive part, while the influence of distortion in the negative part of the quantizer is obtained by multiplication by 2 in (3) and (4). We can write the expression for $D$ in the following form:

$$
\begin{aligned}
D & =2 \int_{0}^{+\infty} x^{2} p(x) d x-2 \Delta \sum_{i=1}^{N / 2}(2 i-1) \int_{(i-1) \Delta}^{i \cdot \Delta} x p(x) d x-2(N-1) \Delta \int_{x_{\max }}^{+\infty} x p(x) d x \\
& +2 \Delta^{2} \sum_{i=1}^{N / 2}(i-1 / 2)^{2} \int_{(i-1) \Delta}^{i \cdot \Delta} p(x) d x+\frac{1}{2}(N-1)^{2} \Delta^{2} \int_{x_{\max }}^{+\infty} p(x) d x .
\end{aligned}
$$

For the Gaussian distribution we have that

and

$$
2 \int_{0}^{+\infty} x^{2} p(x) d x=\sigma^{2}, \int_{a}^{b} x p(x) d x=\frac{\sigma}{\sqrt{2 \pi}}\left(\exp \left(-\frac{a^{2}}{2 \sigma^{2}}\right)-\exp \left(-\frac{b^{2}}{2 \sigma^{2}}\right)\right)
$$

$$
\int_{a}^{b} p(x) d x=\frac{1}{2}\left(\operatorname{erf}\left(\frac{b}{\sqrt{2} \sigma}\right)-\operatorname{erf}\left(\frac{a}{\sqrt{2} \sigma}\right)\right) .
$$

Putting that into (5), the expression for distortion becomes:

$$
D=\sigma^{2}\left[1+\frac{\Delta^{2}}{\sigma^{2}} \frac{(N-1)^{2}}{4}-\sqrt{\frac{2}{\pi}} \frac{\Delta}{\sigma}-2 \frac{\Delta^{2}}{\sigma^{2}} \sum_{i=1}^{N / 2-1} i \cdot \operatorname{erf}\left(\frac{i \cdot \Delta}{\sqrt{2} \sigma}\right)-2 \sqrt{\frac{2}{\pi}} \frac{\Delta}{\sigma} \sum_{i=1}^{N / 2-1} e^{-\frac{i^{2} \Delta^{2}}{2 \sigma^{2}}}\right]
$$

Let us define the relative variance as:

$$
\alpha=10 \cdot \log _{10}\left(\sigma^{2} / \sigma_{0}^{2}\right)[\mathrm{dB}],
$$

which represents the ratio (in $\mathrm{dB}$ ) of the signal variance and the referent variance. If $\sigma^{2}=\sigma_{0}^{2}$, the relative variance is $\alpha=0 \mathrm{~dB}$. Let $\Delta_{0}=\Delta / \sigma_{0}$ denote the normalized quantization stepsize. We have that $\Delta / \sigma=\Delta_{0} \cdot 10^{-\alpha / 20}$. The expression (6) for distortion becomes:

$$
\begin{aligned}
D & =\sigma^{2}\left[1+\Delta_{0}^{2} \cdot 10^{-\frac{\alpha}{10}} \frac{(N-1)^{2}}{4}-\sqrt{\frac{2}{\pi}} \Delta_{0} 10^{-\frac{\alpha}{20}}\right. \\
& \left.-2 \Delta_{0}^{2} \cdot 10^{-\frac{\alpha}{10}} \sum_{i=1}^{N / 2-1} i \cdot \operatorname{erf}\left(\frac{i \cdot \Delta_{0}}{\sqrt{2}} 10^{-\frac{\alpha}{20}}\right)-2 \sqrt{\frac{2}{\pi}} \Delta_{0} 10^{-\frac{\alpha}{20}} \sum_{i=1}^{N / 2-1} e^{-\frac{i^{2} \Delta_{0}^{2}}{2}} 0^{-\frac{\alpha}{10}}\right] .
\end{aligned}
$$


The quality of the quantized signal is defined by the signal-to-noise ratio (SNR), which is defined as:

$$
\mathrm{SNR}[\mathrm{dB}]=10 \cdot \log _{10} \frac{\sigma^{2}}{D}
$$

Based on (8), the expression for SNR becomes:

$$
\begin{aligned}
\mathrm{SNR}[\mathrm{dB}] & =-10 \cdot \log _{10}\left[1+\Delta_{0}^{2} \cdot 10^{-\frac{\alpha}{10}} \frac{(N-1)^{2}}{4}-\sqrt{\frac{2}{\pi}} \Delta_{0} 10^{-\frac{\alpha}{20}}\right. \\
& \left.-2 \Delta_{0}^{2} \cdot 10^{-\frac{\alpha}{10}} \sum_{i=1}^{N / 2-1} i \cdot \operatorname{erf}\left(\frac{i \cdot \Delta_{0}}{\sqrt{2}} 10^{-\frac{\alpha}{20}}\right)-2 \sqrt{\frac{2}{\pi}} \Delta_{0} 10^{-\frac{\alpha}{20}} \sum_{i=1}^{N / 2-1} e^{-\frac{i^{2} \Delta_{0}^{2}}{2} 10^{-\frac{\alpha}{10}}}\right] .
\end{aligned}
$$

SNR is a function of the relative variance $\alpha$ and the normalized stepsize $\Delta_{0}, \mathrm{SNR} \equiv$ $\operatorname{SNR}\left(\alpha, \Delta_{0}\right)$.

The number of quantization levels $N$ is known in advance. To design the uniform quantizer, we firstly have to calculate the normalized stepsize $\Delta_{0}$. It is done by maximizing SNR for the referent variance $\sigma_{0}^{2}$, (i.e. for $\alpha=0 \mathrm{~dB}$ ), i.e. by solving the equation $\left.\frac{\partial \operatorname{SNR}\left(\alpha, \Delta_{0}\right)}{\partial \Delta_{0}}\right|_{\alpha=0}=0$. This maximization is done numerically, in the software package Mathematica. Table 1 gives values of $\Delta_{0}$ for the different values of the number of quantization levels $N$. Values of the maximal SNR (obtained for $\alpha=0 \mathrm{~dB}$ ) are also presented in Table 1. The quantization stepsize $\Delta$ is calculated as $\Delta=\Delta_{0} \cdot \sigma_{0}$; using (2) we can calculate all thresholds and representation levels, which completes the design of the uniform quantizer.

Table 1 Parameters and performances of the uniform quantizer

\begin{tabular}{cccc}
\hline$N$ & $\Delta_{0}$ & $x_{\max }$ & $S N R_{\max }[\mathrm{dB}]$ \\
\hline 2 & 1.5958 & 1.5958 & 4.40 \\
4 & 0.9957 & 1.9914 & 9.25 \\
8 & 0.5860 & 2.3441 & 14.27 \\
16 & 0.3352 & 2.6816 & 19.38 \\
32 & 0.1881 & 3.0102 & 24.57 \\
64 & 0.1041 & 3.3300 & 29.83 \\
128 & 0.0569 & 3.6395 & 35.17 \\
256 & 0.0308 & 3.9376 & 40.57 \\
\hline
\end{tabular}

If we put the calculated value for $\Delta_{0}$ in (10), SNR becomes a function of only one parameter $\alpha$, i.e., $\operatorname{SNR} \equiv \operatorname{SNR}(\alpha)$. Fig. 1 shows $\operatorname{SNR}(\alpha)$ for different values of the number of quantization levels $N$. We can see that SNR has the highest value for $\alpha=0 \mathrm{~dB}$ (i.e., when the signal variance is equal to the referent variance), while SNR decreases when the distance between the signal variance and the referent variance increases.

Each signal sample is coded with $\log _{2} N$ bits, hence the average bit-rate of the PCM system is $R_{\mathrm{PCM}}=\log _{2} N$ [bps]. 


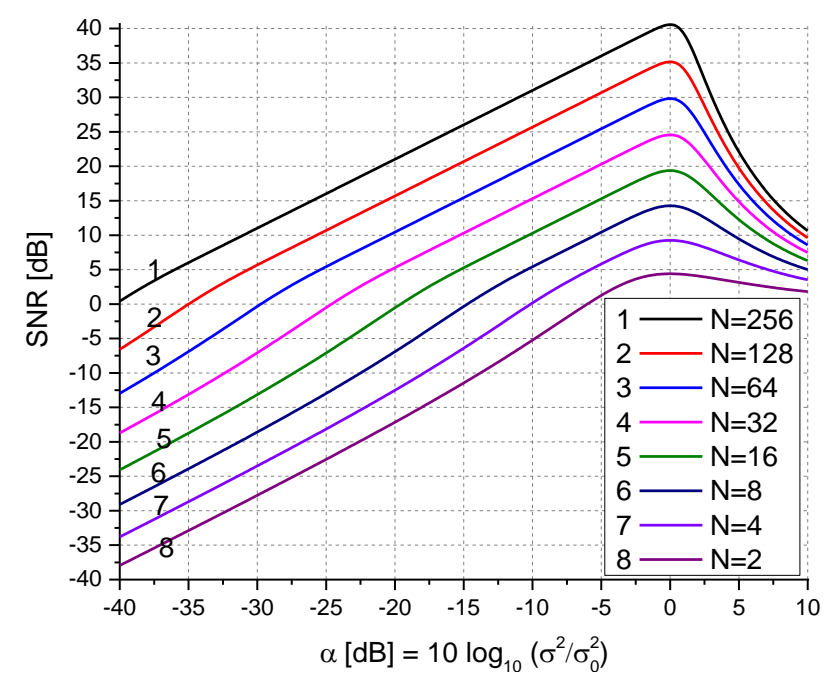

Fig. $1 \operatorname{SNR}(\alpha)$ of the uniform quantizer for different values of $N$

Since the signal variance $\sigma^{2}$ can vary in the very wide range, it can significantly differ from the referent variance $\sigma_{0}^{2}$ for which the quantizer is designed, which can produce significant degradation of performances. As a consequence of that, we have to use the uniform quantizer with a large number of levels to achieve required performances in the whole range of the signal variance.

\subsection{APCM system}

When the signal variance $\sigma^{2}$ varies in a wide range, adaptive quantization can be performed, with the aim to adapt the measurement signal to the quantizer. In this way we obtain the APCM (adaptive PCM) system, whose block diagram is shown in Fig. 2.

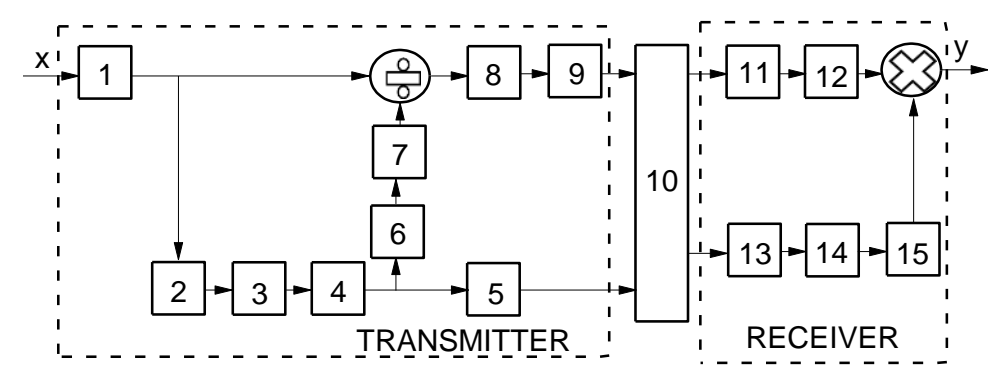

Fig. 2 The block diagram of the APCM system (1: buffer, 2: calculation of the variance $\sigma^{2}$, 3: calculation of the relative variance $\alpha, 4: \log$-uniform quantizer for quantization of $\alpha$, 5: coder for $\alpha, 6$ : dequantization of $\alpha, 7$ : calculation of $\sigma^{*} / \sigma_{0}, 8: N$-level fixed uniform quantizer designed for the referent variance, 9: coder, 10: channel, 11: decoder, 12: dequantizer, 13: decoder for $\alpha, 14$ : dequantizer for $\alpha, 15$ : calculation of $\sigma^{*} / \sigma_{0}$ 
The adaptation is done frame-by-frame. In the buffer (block 1 in Figure 2), frames of $M$ signal samples are formed. The variance of each frame is calculated in block 2 as $\sigma^{2}=\left(\sum_{i=1}^{M} x_{i}^{2}\right) / M$, where $x_{i}$ denote signal samples within the frame. If each sample in the frame would be divided with $\sigma / \sigma_{0}$, the variance of the frame would be $\left(\sum_{i=1}^{M}\left(x_{i} /\left(\sigma / \sigma_{0}\right)\right)^{2}\right) / M=\left(\sigma_{0}^{2} / \sigma^{2}\right) \cdot\left(\sum_{i=1}^{M} x_{i}^{2}\right) / M=\left(\sigma_{0}^{2} / \sigma^{2}\right) \cdot \sigma^{2}=\sigma_{0}^{2}$, meaning that the frame would be fully adapted to the quantizer. However, this idea should be modified because the information about the frame variance should be transmitted to the receiver, so the frame variance must be quantized, which is best done using a log-uniform quantizer (i.e. by performing a uniform quantization of the relative variance $\alpha$ in the logarithmic domain). This is done in the following way. Firstly, the relative variance $\alpha$ of the frame is calculated in block 3 using (7), and then the uniform quantization of $\alpha$ is performed in block 4 by dividing the range of interest for $\alpha$ uniformly into $K$ intervals of the width $\Delta_{\alpha}$. There are the following $K$ intervals for the quantization of $\alpha$ : $\left(\alpha_{a}+k \Delta \alpha, \alpha_{b}+k \Delta_{\alpha}\right)$, $k=-m, \ldots, 0, \ldots, n, m+n+1=K$, where $\alpha_{a}<0 \mathrm{~dB}<\alpha_{b}, \alpha_{b}-\alpha_{a}=\Delta_{\alpha}$. Quantized relative variance is coded with $\log _{2} K$ bits in the coder (block 5) and it is sent through the channel to the receiver.

In block 6 , dequantization of the relative variance $\alpha$ is performed, obtaining the representation level $\alpha^{*}$ of the relative variance. For the interval $\left(\alpha_{a}, \alpha_{b}\right)$, the representation level is $\alpha_{0}{ }^{*}=0 \mathrm{~dB}$ since this value provides the maximal SNR; for the $k$-th interval $\left(\alpha_{a}+k \Delta_{\alpha}, \alpha_{b}+k \Delta_{\alpha}\right)$, the representation level is $\alpha^{*}=\alpha_{k}^{*}=\alpha_{0}^{*}+k \cdot \Delta_{\alpha}=k \cdot \Delta_{\alpha} \mathrm{dB}$. For the obtained $\alpha^{*}$, we calculate $\sigma^{*} / \sigma_{0}$ in block 7, where $\sigma^{*}=\sigma_{0} \cdot 10^{\alpha^{*} / 20}$.

All samples $x_{i}$ in the frame are divided with $\sigma^{*} / \sigma_{0}$, obtaining the adapted samples $x_{i}^{a d}=x_{i} /\left(\sigma^{*} / \sigma_{0}\right), i=1, \ldots, M$. Each adapted sample $x_{i}^{a d}$ is firstly quantized by the fixed quantifier $Q_{\text {fix }}$ (block 8) with $N$ levels, designed for the referent variance $\sigma_{0}^{2}$; after that, it is coded with $\log _{2} N$ bits in the coder (block 9) and sent through the channel (block 10) to the receiver.

The variance of the frame of adapted samples is $\left(\sigma^{a d}\right)^{2}=\left(\sum_{i=1}^{M}\left(x_{i}^{a d}\right)^{2}\right) / M$, $=\left(\sum_{i=1}^{M}\left(x_{i} /\left(\sigma^{*} / \sigma_{0}\right)\right)^{2}\right) / M=\left(\sigma^{*} / \sigma_{0}\right)^{2} \cdot\left(\sum_{i=1}^{M} x_{i}^{2}\right) / M=\left(\sigma_{0} \cdot \sigma / \sigma^{*}\right)^{2}$, hence the relative variance of the frame of adapted samples is:

$$
\alpha^{a d}=10 \log _{10} \frac{\left(\sigma^{a d}\right)^{2}}{\sigma_{0}^{2}}=20 \log _{10} \frac{\sigma / \sigma_{0}}{\sigma^{*} / \sigma_{0}}=10 \log _{10} \frac{\sigma^{2}}{\sigma_{0}^{2}}-10 \log _{10} \frac{\left(\sigma^{*}\right)^{2}}{\sigma_{0}^{2}}=\alpha-\alpha^{*}
$$

If $\alpha \in\left(\alpha_{a}+k \Delta_{\alpha}, \alpha_{b}+k \Delta_{\alpha}\right)$, we have that $\alpha^{*}=k \cdot \Delta_{\alpha} \mathrm{dB}$, hence it follows that $\alpha^{a d} \in\left(\alpha_{a}, \alpha_{b}\right)$. Therefore, the variance of the adapted signal is located in a narrow range $\left(\alpha_{a}, \alpha_{b}\right)$ around $0 \mathrm{~dB}$, i.e., it is very close to the referent variance $\alpha=0 \mathrm{~dB}$ for which the fixed quantizer $Q_{\text {fix }}$ is designed. In this way, the adjustment of the signal to the fixed quantizer $Q_{\text {fix }}$ has been achieved.

In the receiver, decoding and dequantization of the adapted signal samples are performed in blocks 11 and 12, obtaining the representative values for the adapted samples $y_{i}^{a d}$. Decoding and dequantization of the relative variance are also performed, in blocks 13 and 14, obtaining the representation value of the relative variance $\alpha^{*}$. Then, 
$\sigma^{*} / \sigma_{0}$ is calculated in the block 15 in the same way as in block 7. At the end, the reconstructed value of the signal sample is calculated as $y_{i}=y_{i}^{a d} \cdot\left(\sigma^{*} / \sigma_{0}\right)$.

At the input of the adaptive quantizer we have signal samples $x_{i}$ whose variance is $\sigma^{2}$ (i.e. the relative variance is $\alpha$ ), while at the output we obtain the reconstructed samples $y_{i}$, $i=1, \ldots, M$. Hence, we can define the distortion of the adaptive quantizer as $D^{a d}=\left(\sum_{i=1}^{M}\left(x_{i}-y_{i}\right)^{2}\right) / M$ and the signal-to-noise ratio of the adaptive quantizer as $S N R^{a d}=10 \cdot \log _{10}\left(\sigma^{2} / D^{a d}\right)$. As we know, signal-to-noise ratio is a function of the relative variance, hence we have that $S N R^{a d} \equiv S N R^{a d}(\alpha)$.

At the input of the fixed quantizer we have adapted samples $x_{i}^{a d}$ whose variance is $\left(\sigma^{a d}\right)^{2}$ and the relative variance is $\alpha^{a d}$, while at the output we obtain the reconstructed adapted samples $y_{i}^{a d}, i=1, \ldots, M$. Hence, we can define the distortion of the fixed quantizer as $D^{f i x}=\left(\sum_{i=1}^{M}\left(x_{i}^{a d}-y_{i}^{a d}\right)^{2}\right) / M$ and the signal-to-noise ratio of the fixed quantizer as $S N R^{f i x}=10 \cdot \log _{10}\left(\left(\sigma^{a d}\right)^{2} / D^{f i x}\right)$. Since signal-to-noise ratio is a function of the relative variance, we have that $S N R^{f i x} \equiv S N R^{f i x}\left(\alpha^{a d}\right)$. It follows that $D^{f i x}=$ $\left(\sum_{i=1}^{M}\left(\left(x_{i}-y_{i}\right) /\left(\sigma^{*} / \sigma_{0}\right)\right)^{2}\right) / M=\left(\sigma_{0} / \sigma^{*}\right)^{2}\left(\sum_{i=1}^{\bar{M}}\left(x_{i}-y_{i}\right)^{2}\right) / M=\left(\sigma_{0} / \sigma^{*}\right)^{2} D^{a d}$. We can obtain that $D^{a d}=\left(\sigma^{*} / \sigma_{0}\right)^{2} D^{f i x}$. Finally, we have that:

$$
\begin{aligned}
\operatorname{SNR}^{a d}(\alpha) & =10 \cdot \log _{10} \frac{\sigma^{2}}{D^{a d}}=10 \cdot \log _{10} \frac{\sigma^{2}}{\left(\left(\sigma^{*}\right)^{2} / \sigma_{0}^{2}\right) \cdot D^{f i x}}=10 \cdot \log _{10} \frac{\left(\sigma_{0} \cdot \sigma / \sigma^{*}\right)^{2}}{D^{f i x}} \\
& =10 \cdot \log _{10} \frac{\left(\sigma^{a d}\right)^{2}}{D^{f i x}}=S N R^{f i x}\left(\alpha^{a d}\right) .
\end{aligned}
$$

From (11) and (12), it follows that $\operatorname{SNR}^{a d}(\alpha)=\operatorname{SNR}^{f i x}\left(\alpha-\alpha^{*}\right)$.

If $\alpha$ belongs to some arbitrary interval $\alpha \in\left(\alpha_{a}+k \Delta_{\alpha}, \alpha_{b}+k \Delta_{\alpha}\right), k=-m, \ldots, 0, \ldots, n$, knowing that $\alpha^{*}=k \cdot \Delta_{\alpha}$, we obtain that

$$
\operatorname{SNR}^{a d}(\alpha)=\operatorname{SNR}^{f i x}\left(\alpha-k \cdot \Delta_{\alpha}\right) \text { for } \alpha \in\left(\alpha_{a}+k \Delta_{\alpha}, \alpha_{b}+k \Delta_{\alpha}\right), k=-m, \ldots, 0, \ldots, n .
$$

Since $\alpha-k \cdot \Delta_{\alpha} \in\left(\alpha_{a}, \alpha_{b}\right)$, we can conclude that $\operatorname{SNR}^{a d}(\alpha)$ is obtained by the periodic repetition (with the period $\left.\Delta_{\alpha}\right)$ of the portion of $S N R^{f i x}$ in the range $\left(\alpha_{a}, \alpha_{b}\right)$ around 0 , that is shown in Fig. 3. We can see that the adaptive quantizer has almost the constant SNR in the very wide range of the signal variance.

The average bit-rate of the APCM system is $R_{A P C M}=\log _{2} N+\left(\log _{2} K\right) / M$ [bps], where the last term denotes additional information about the relative variance. For large values of $M$ (which is always true in practice), it follows that $R_{A P C M}=\log _{2} N$ [bps].

\subsection{DPCM system}

One of the important features of measurement signals is that they are correlated. We can define the correlation coefficient as

$$
\rho=\left|\frac{\sum_{i=1}^{M-1} x_{i} \cdot x_{i+1}}{\sum_{i=1}^{M} x_{i}^{2}}\right|,
$$


where $M$ denotes the number of samples in the signal (or the number of samples in the frame if the correlation coefficient is calculated frame-by-frame). In highly correlated signals, adjacent samples usually have very similar values; hence, values of the differences of adjacent samples are usually much smaller than values of the signal samples. It leads us to the idea to use differential coding for the analog-to-digital conversion and transmission of highly correlated signals: instead of the original signal samples, the differences of adjacent samples are quantized, coded and transmitted. Such a system is called a DPCM (differential PCM) system. Since the range of samples' differences is much smaller than the range of the original signal, we can use a quantizer with a substantially smaller number of levels (i.e. we can represent the signal with a substantially smaller number of bits), preserving the same quality.

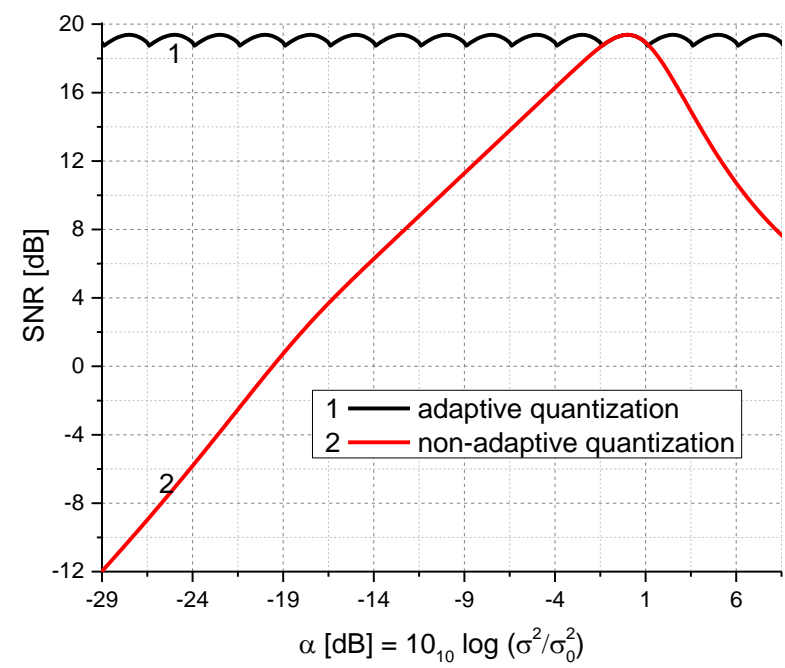

Fig. $3 \operatorname{SNR}(\alpha)$ of the adaptive and non-adaptive uniform quantizers for $N=16$ levels

We will describe the DPCM system, whose block diagram is shown in Fig. 4.

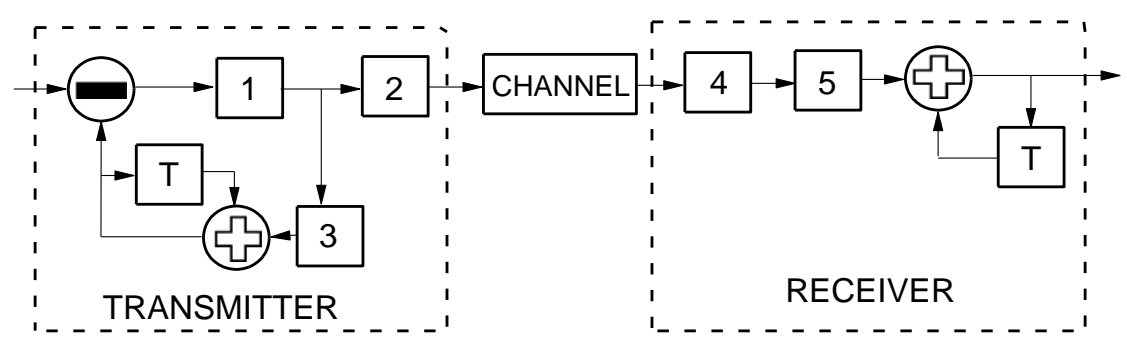

Fig. 4 The block diagram of the DPCM system

(1: quantizer, 2: coder, 3: dequantizer, 4: decoder, 5: dequantizer, T: time delay) 
Let $x_{i}$ represent the $i$-th sample of the original signal and $y_{i}$ denote the $i$ - th sample of the reconstructed signal, $i=1, \ldots, M$. It is important to transmit the first signal sample $x_{1}$ as accurately as possible (to minimize the error propagation), hence $x_{1}$ is encoded with a large number of bits $r_{1}$ (e.g. $r_{1}=24$ bits). Therefore, the reconstructed sample $y_{1}$ is almost the same as the original sample $x_{1}$, i.e. $y_{1} \approx x_{1}$. The difference $\Delta x_{i}=x_{i+1}-y_{i}$ ( $i=1, \ldots, M-1)$ is formed as the difference of the $(i+1)$-th signal sample and the $i$-th sample of the reconstructed signal. The difference $\Delta x_{i}$ is quantized using the uniform quantizer (block 1 in Fig. 4) with $N_{\Delta}$ levels designed for the reference variance $\sigma_{\Delta 0}^{2}$ and coded in the coder (block 2) with $\log _{2} N_{\Delta}$ bits. Dequantization of the difference is done in block 3, obtaining the representation value of the difference $\Delta y_{i}$, which is used to calculate $y_{i+1}$ as $y_{i+1}=y_{i}+\Delta y_{i}$, that we need for the calculation of the next difference.

In the receiver, firstly the decoding and dequantization of the difference is done (in blocks 4 and 5) and the representation value of the difference $\Delta y_{i}$ is obtained. Finally, the reconstructed sample is obtained as $y_{i+1}=y_{i}+\Delta y_{i}$. Just to say, it is not a good idea to use differences $\Delta x_{i}=x_{i+1}-x_{i}$, since $x_{1}$ is not available to the receiver, hence the accurate reconstruction cannot be done.

It is known that the variance of differences $\sigma_{\Delta}^{2}$ can be calculated based on the signal variance $\sigma^{2}$ as

$$
\sigma_{\Delta}^{2}=\sigma^{2} \cdot 2(1-\rho)
$$

Higher values of the correlation coefficient $\rho$ give lower values of the variance of the differences.

Let $D=\left(\sum_{i=1}^{M}\left(x_{i}-y_{i}\right)^{2}\right) / M$ denote the distortion of the digitalization of the signal $x$ and $D_{\Delta}=\left(\sum_{i=1}^{M-1}\left(\Delta x_{i}-\Delta y_{i}\right)^{2}\right) / M$ denote the distortion of the quantization of differences. Since $y_{1} \approx x_{1}$, it follows that $D=\left(\sum_{i=1}^{M-1}\left(x_{i+1}-y_{i+1}\right)^{2}\right) / M$. Further, we can obtain that $D=\left(\sum_{i=1}^{M-1}\left(x_{i+1}-y_{i+1}\right)^{2}\right) / M=\left(\sum_{i=1}^{M-1}\left(y_{i}+\Delta x_{i}-\left(y_{i}+\Delta y_{i}\right)\right)^{2}\right) / M=\left(\sum_{i=1}^{M-1}\left(\Delta x_{i}-\Delta y_{i}\right)^{2}\right) / M=D_{\Delta}$, which means that the distortion of the digitalization of the signal $x$ is equal to the distortion of the quantization of differences.

Let $\mathrm{SNR}=10 \cdot \log _{10}\left(\sigma^{2} / D\right)$ denote the signal-to-noise ratio of the digitalization of the signal $x$ and $\mathrm{SNR}_{\Delta}=10 \cdot \log _{10}\left(\sigma_{\Delta}^{2} / D_{\Delta}\right)$ denote the signal-to-noise ratio of the quantization of differences $\Delta x_{i}$. Then, we have that:

$$
\begin{aligned}
\mathrm{SNR} & =10 \cdot \log _{10} \frac{\sigma^{2}}{D}=10 \cdot \log _{10} \frac{\sigma^{2}}{D_{\Delta}}=10 \cdot \log _{10} \frac{\sigma_{\Delta}^{2}}{2(1-\rho) D_{\Delta}} \\
& =10 \cdot \log _{10} \frac{\sigma_{\Delta}^{2}}{D_{\Delta}}+10 \cdot \log _{10} \frac{1}{2(1-\rho)}=\mathrm{SNR}_{\Delta}+10 \cdot \log _{10} \frac{1}{2(1-\rho)} .
\end{aligned}
$$

The term $G=10 \cdot \log _{10} \frac{1}{2(1-\rho)}[\mathrm{dB}]$ denotes the gain due to the correlation. The values of $G$ for different values of the correlation coefficient $\rho$ are given in Table 2 . 
Table 2 Values of the gain $G[\mathrm{~dB}]$ for different values of the correlation coefficient $\rho$

\begin{tabular}{cccccccc}
\hline$\rho$ & $G$ & $\rho$ & $G$ & $\rho$ & $G$ & $\rho$ & $G$ \\
\hline 0.968 & 11.94 & 0.976 & 13.19 & 0.984 & 14.95 & 0.992 & 17.96 \\
0.969 & 12.08 & 0.977 & 13.37 & 0.985 & 15.23 & 0.993 & 18.54 \\
0.970 & 12.22 & 0.978 & 13.57 & 0.986 & 15.53 & 0.994 & 19.21 \\
0.971 & 12.37 & 0.979 & 13.77 & 0.987 & 15.85 & 0.995 & 20.00 \\
0.972 & 12.52 & 0.980 & 13.98 & 0.988 & 16.20 & 0.996 & 20.97 \\
0.973 & 12.68 & 0.981 & 14.20 & 0.989 & 16.58 & 0.997 & 22.22 \\
0.974 & 12.84 & 0.982 & 14.44 & 0.990 & 16.99 & 0.998 & 23.98 \\
0.975 & 13.01 & 0.983 & 14.69 & 0.991 & 17.45 & 0.999 & 26.99 \\
\hline
\end{tabular}

From equation (16) it follows that $\mathrm{SNR}_{\Delta}=\mathrm{SNR}-G[\mathrm{~dB}]$. It means that the required signal-to-noise ratio for the quantization of differences $\Delta x_{i}$ is for $G \mathrm{~dB}$ smaller than the required signal-to-noise ratio for the quantization of the signal samples. Hence, the differences can be quantized with smaller number of quantization levels and coded with smaller number of bits. In other words, DPCM system requires smaller number of bits to achieve the same signal quality, compared to PCM system.

To recall, the first signal sample $x_{1}$ is coded with $r_{1}$ bits, while each of $M-1$ differences is coded with $\log _{2} N_{\Delta}$ bits. Hence, the average bit-rate of DPCM system is $R_{D P C M}=\left((M-1) \cdot \log _{2} N_{\Delta}+r_{1}\right) / M=\log _{2} N_{\Delta}+\left(r_{1}-\log _{2} N_{\Delta}\right) / M$ [bps]. For large values of $M$ (which is always true in practice), it follows that $R_{D P C M}=\log _{2} N_{\Delta}$ [bps].

\subsection{ADPCM system}

The ADPCM system is based on the DPCM system (i.e. on the quantization and coding of the differences of consecutive signal samples), but it includes double adaptation (on the variance and on the correlation), to solve the problem of significant variations of variance and correlation coefficient in time. The block diagram of the ADPCM system is shown in Fig. 5.

The ADPCM system works as follows. Frames of $M$ signal samples are formed in the buffer (block 1 in Fig. 5) since the adaptation is done on the frame-by-frame basis. The variance of samples $\sigma^{2}$ of each frame is calculated in the block 2 and the relative variance $\alpha$ of the frame is calculated in the block 3 according to (7), in relation to some referent variance $\sigma_{0}^{2} . K$-level uniform quantization is performed in the block 4 in the manner described in section 2.2. Quantized relative variance is coded in the coder (block 5) with $\log _{2} K$ bits and sent through the channel to the receiver. The correlation coefficient $\rho$ of the signal frame is calculated in the block 6 according to formula (14). $P$-level uniform quantization of the correlation coefficient $\rho$ is done in the block 7, in the following way: the minimal and maximal values $\rho_{\min }$ and $\rho_{\max }$ of the correlation coefficient $\rho$ are selected; the range $\left(\rho_{\min }, \rho_{\max }\right)$ is divided into $P$ quantization intervals of the width $\Delta_{\rho}=\left(\rho_{\max }-\rho_{\min }\right) / P$; the thresholds for the uniform quantization of $\rho$ are defined as $\rho_{\min }+i \cdot \Delta_{\rho}, i=0, \ldots, P$, while the representation levels are defined as $\rho_{\min }+(i-1 / 2) \cdot \Delta_{\rho}, i=1, \ldots, P$. Quantized correlation coefficient is coded in the coder (block 8 ) with $\log _{2} P$ bits and sent through the channel. The dequantization of the relative variance is performed in block 9 obtaining the representation value $\alpha^{*}$, while the dequantization of the 
correlation coefficient is performed in block 10 obtaining the representation value $\rho^{*}$. The ratio $\sigma_{\Delta} / \sigma_{\Delta 0}$ is calculated in block 11 , where $\sigma_{\Delta}^{2}$ denotes the variance of differences of consecutive samples within the frame; it is calculated as $\sigma_{\Delta}^{2}=\left(\sigma^{*}\right)^{2} 2\left(1-\rho^{*}\right)$, according to (15), where $\sigma^{*}=\sigma_{0} \cdot 10^{\alpha^{*} / 20}$. As we will see soon, $\sigma_{\Delta 0}^{2}$ is the referent variance for which the quantizer for adapted differences (block 12) is designed.

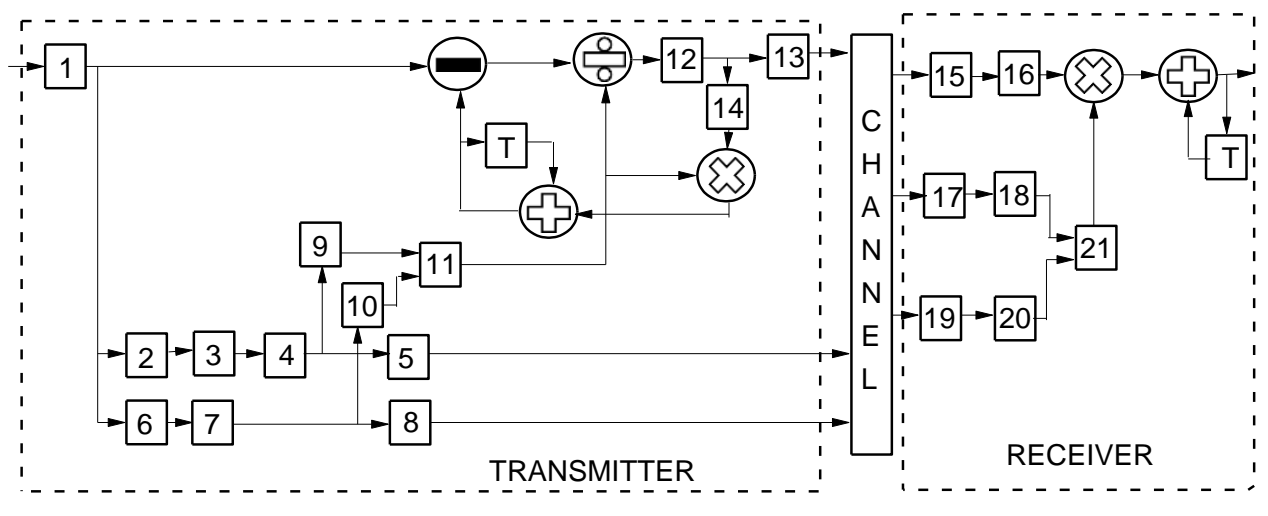

Fig. 5 Structure of fuzzy controller (1: buffer, 2: calculation of $\sigma^{2}, 3$ : calculation of $\alpha$, 4: $\log$ uniform quantization of $\alpha, 5$ : coder for $\alpha, 6$ : calculation of $\rho, 7$ : quantizer for $\rho, 8$ : coder for $\rho$, 9: dequantizer for $\alpha, 10$ : dequantizer for $\rho$, 11: calculation of $\sigma_{\Delta} / \sigma_{\Delta 0}$, 12: quantizer for adapted differences $\Delta x_{i}^{a d}, 13$ : coder, 14: dequantizer, 15: decoder for $\Delta x_{i}^{a d}, 16$ : dequantizer for $\Delta x_{i}^{a d}, 17$ : decoder for $\alpha, 18$ : dequantizer for $\alpha, 19$ : decoder for $\rho, 20$ : dequantizer for $\rho, 21$ : calculation of $\sigma_{\Delta} / \sigma_{\Delta 0}$ )

The first sample in each frame is transmitted as accurate as possible with large number of bits $r_{1}$; hence, the reconstructed sample $y_{1}$ is almost the same as the original sample $x_{1}$, i.e. $y_{1} \approx x_{1}$. The differences $\Delta x_{i}=x_{i+1}-y_{i}, i=1, \ldots, M-1$ are formed and divided by $\sigma_{\Delta} / \sigma_{\Delta 0}$, obtaining the adapted differences $\Delta x_{i}^{a d}=\Delta x_{i} /\left(\sigma_{\Delta} / \sigma_{\Delta 0}\right)$, that are quantized with the $N_{\Delta}$-level uniform quantizer (block 12) designed for the referent variance $\sigma_{\Delta 0}^{2}$, coded with $\log _{2} N_{\Delta}$ bits in the encoder (block 13) and sent through the channel. Dequantization is done in block 14, obtaining reconstructed adapted differences $\Delta y_{i}^{a d}$, that are multiplied with $\sigma_{\Delta} / \sigma_{\Delta 0}$ to obtain $\Delta y_{i}$, that are then added to $7 y_{i}$, to finally obtain $y_{i+1}$.

In the receiver, the following actions are performed: decoding and dequantization of the adapted differences (blocks 15 and 16), obtaining reconstructed adapted differences $\Delta y_{i}^{a d}$; decoding and dequantization of relative variance (blocks 17 and 18), obtaining the representation value of the relative variance $\alpha^{*}$; decoding and dequantization of the correlation coefficient (blocks 19 and 20), obtaining the representation value of the correlation coefficient $\rho^{*}$. In block 21, $\sigma_{\Delta} / \sigma_{\Delta 0}$ is calculated, where $\sigma_{\Delta}=\sigma_{0} \cdot 10^{\alpha^{*} / 20} \cdot \sqrt{2\left(1-\rho^{*}\right)}$. Then $\Delta y_{i}=\Delta y_{i}^{a d} \cdot \sigma_{\Delta} / \sigma_{\Delta 0}$ is calculated; finally, the reconstructed signal sample $y_{i+1}=y_{i}+\Delta y_{i}$ is obtained. 
To summarize. The first signal sample of each frame $x_{1}$ is coded with $r_{1}$ bits, then each of $M-1$ differences is coded with $\log _{2} N_{\Delta}$ bits, the quantized variance and the quantized correlation coefficient of the frame are encoded with $\log _{2} K$ and $\log _{2} P$ bits. The average bit-rate of the ADPCM system is $R_{A D P C M}=\left((M-1) \cdot \log _{2} N_{\Delta}+r_{1}+\log _{2} K+\log _{2} P\right) / M$ $=\log _{2} N_{\Delta}+\left(r_{1}-\log _{2} N_{\Delta}+\log _{2} K+\log _{2} P\right) / M$ [bps]. For large $M$ (which is always true in practice), it follows that $R_{A D P C M}=\log _{2} N_{\Delta}$ [bps].

\section{DESIGN OF FOUR ADC SYSTEMS FOR VIBRATION SIGNALS}

In this section, four ADC systems (PCM, APCM, DPCM and ADPCM) will be designed for vibration signals. Firstly, 20 vibration signals from the referent signal database RealVibrations [12] will be analized to determine their characteristics. Then, four ADC systems will be designed, based on the obtained characteristics of the vibration signals. Finally, to examine performances of the designed ADC systems, they will be applied for the analog-to-digital conversion of the considered vibration signals.

\subsection{Analysis of vibration signals}

We analyze 20 vibration signals from the referent database RealVibrations [12]. Since these signals are very long (each of them contains several hundred thousand samples), each signal is divided into frames of length of $M=1000$ samples. For each frame, the following parameters are calculated: $\sigma, \rho$ and $\sigma_{\Delta}$ (where $\sigma^{2}$ represents the frame variance, $\rho$ is the correlation coefficient of the samples in the frame and $\sigma_{\Delta}^{2}$ represents the variance of differences between consecutive samples in the frame). The values of these parameters vary from frame to frame within one signal. In Table 3 , minimal and maximal values of these parameters are presented: $\left(\sigma_{\min }, \sigma_{\max }\right),\left(\rho_{\min }, \rho_{\max }\right)$ and $\left(\sigma_{\Delta \min }, \sigma_{\Delta \max }\right)$, for each signal. These bands do not include $10 \%$ of the lowest and $10 \%$ of the highest values, since extremely small and large values that occur sporadically do not need to be taken into account during the design of the ADC system.

We see that the parameters $\sigma, \rho$ and $\sigma_{\Delta}$ vary quite considerably, both within one signal and from one signal to another. Observed for all signals, $\sigma$ varies from 0.0095 to 0.2744 , hence the variance $\sigma^{2}$ is placed in the range of the width of $10 \log _{10}\left(0.2744^{2} / 0.0095^{2}\right)=$ $29.21 \mathrm{~dB}$ in the logarithmic domain; $\rho$ is placed in the range from 0.9028 to $0.9991 ; \sigma_{\Delta}$ varies from 0.0025 to 0.0233 , hence $\sigma_{\Delta}^{2}$ occupies the range of $19.39 \mathrm{~dB}$ in the logarithmic domain.

We can see that the variance $\sigma^{2}$ of the considered vibration signals varies in the very wide range of almost $30 \mathrm{~dB}$. The variance of differences of consecutive samples $\sigma_{\Delta}^{2}$ takes much smaller values than $\sigma^{2}$ and occupies narrower range (of about $20 \mathrm{~dB}$ ). Also, we can see that the vibration signals are highly correlated. 
Table 3 Ranges of parameters values of vibration signals

\begin{tabular}{cccc}
\hline Signals & $\left(\sigma_{\min }, \sigma_{\max }\right)$ & $\left(\rho_{\min }, \rho_{\max }\right)$ & $\left(\sigma_{\Delta \min }, \sigma_{\Delta \max }\right)$ \\
\hline airconditioner X & $0.0381-0.0554$ & $0.9720-0.9918$ & $0.0061-0.0095$ \\
airconditioner Z & $0.1513-0.2602$ & $0.9929-0.9974$ & $0.0141-0.0174$ \\
car X & $0.0395-0.1169$ & $0.9875-0.9977$ & $0.0054-0.0070$ \\
car Y & $0.0417-0.2306$ & $0.9894-0.9987$ & $0.0054-0.0064$ \\
car1 Y & $0.0208-0.1087$ & $0.9827-0.9985$ & $0.0035-0.0045$ \\
car1 Z & $0.0282-0.2665$ & $0.9231-0.9989$ & $0.0076-0.0114$ \\
car2 X & $0.0190-0.1248$ & $0.9842-0.9986$ & $0.0032-0.0044$ \\
car2 Y & $0.0180-0.1788$ & $0.9782-0.9989$ & $0.0035-0.0042$ \\
car3 Y & $0.0176-0.1618$ & $0.9827-0.9988$ & $0.0027-0.0040$ \\
car3 Z & $0.0142-0.2356$ & $0.9459-0.9991$ & $0.0040-0.0068$ \\
car4 Y & $0.0309-0.2021$ & $0.9842-0.9988$ & $0.0039-0.0130$ \\
car4 Z & $0.0457-0.2744$ & $0.9749-0.9983$ & $0.0086-0.0233$ \\
car5 X & $0.0258-0.1442$ & $0.9118-0.9984$ & $0.0034-0.0219$ \\
car5 Y & $0.0286-0.1499$ & $0.9028-0.9984$ & $0.0032-0.0217$ \\
microwave_oven X & $0.0226-0.0323$ & $0.9885-0.9941$ & $0.0031-0.0035$ \\
microwave_oven Y & $0.0146-0.0257$ & $0.9748-0.9920$ & $0.0030-0.0037$ \\
train (seat object free) X & $0.0200-0.0361$ & $0.9893-0.9964$ & $0.0026-0.0028$ \\
train (seat object free) Z & $0.0276-0.0511$ & $0.9937-0.9981$ & $0.0026-0.0028$ \\
train (seat) X & $0.0095-0.0354$ & $0.9610-0.9964$ & $0.0025-0.0029$ \\
train (seat) Z & $0.0195-0.1019$ & $0.9897-0.9987$ & $0.0025-0.0028$ \\
\hline
\end{tabular}

\subsection{Design of ADC systems for vibration signals}

Based on the theoretical analysis from Chapter 2, the design of four ADC systems for the digitalization of the vibration signals is performed. During design, the aim is to achieve SNR higher than some minimal acceptable value $\mathrm{SNR}_{\text {min }}$, for all considered vibration signals (in this case we took the value $\mathrm{SNR}_{\min }=23 \mathrm{~dB}$ ). Taking into account the vibration signal analysis in section 3.1, the following parameters of these four ADC systems are defined: PCM: $(N=256$, $\left.\sigma_{0}=0.16\right)$; APCM: $\left(N=32, \sigma_{0}=0.12, M=1000, K=16, m=12, n=3, \alpha_{a}=-1.5 \mathrm{~dB}, \alpha_{b}=1\right.$ $\left.\mathrm{dB}, \Delta_{\alpha}=2.5 \mathrm{~dB}\right)$; DPCM: $\left(N_{\Delta}=16, \sigma_{\Delta 0}=0.014, M=1000, r_{1}=24\right)$; ADPCM: $\left(N_{\Delta}=4, \sigma_{\Delta 0}=\right.$ $0.014, M=1000, r_{1}=24, K=16, m=10, n=5, \alpha_{a}=-1 \mathrm{~dB}, \alpha_{b}=0.5 \mathrm{~dB}, \Delta_{\alpha}=1.5 \mathrm{~dB}, P=32$, $\rho_{\text {min }}=0.903, \rho_{\max }=0.999, \sigma_{0}=0.12$ ).

Designed four ADC systems are applied for digitalization of 20 considered vibration signals. Table 4 shows obtained numerical results. 
Table 4 Experimental results

\begin{tabular}{lcccc}
\hline \multirow{2}{*}{ Signals } & \multicolumn{4}{c}{ SNR [dB] } \\
\cline { 2 - 5 } & PCM & APCM & DPCM & ADPCM \\
\hline airconditioner X & 30.18 & 24.79 & 30.56 & 23.65 \\
airconditioner Z & 35.20 & 25.04 & 34.46 & 26.16 \\
car X & 34.94 & 24.89 & 35.36 & 25.25 \\
car Y & 40.24 & 25.06 & 40.66 & 33.33 \\
car1 Y & 34.93 & 25.06 & 35.36 & 33.18 \\
car1 Z & 42.59 & 24.65 & 42.99 & 33.30 \\
car2 X & 35.13 & 25.51 & 34.95 & 30.34 \\
car2 Y & 38.47 & 24.75 & 38.88 & 35.48 \\
car3 Y & 35.96 & 24.89 & 36.38 & 34.44 \\
car3 Z & 41.48 & 25.17 & 41.91 & 35.34 \\
car4 Y & 36.04 & 25.40 & 37.21 & 26.83 \\
car4 Z & 38.86 & 25.27 & 28.57 & 27.90 \\
car5 X & 36.28 & 24.96 & 30.80 & 24.57 \\
car5 Y & 36.30 & 24.97 & 32.63 & 24.92 \\
microwave_oven X & 25.81 & 25.04 & 26.24 & 27.01 \\
microwave_oven Y & 23.25 & 24.92 & 23.68 & 24.48 \\
train (seat object free) X & 26.03 & 24.97 & 26.46 & 29.18 \\
train (seat object free) Z & 29.01 & 24.97 & 29.44 & 31.78 \\
train (seat) X & 24.32 & 24.85 & 24.73 & 25.54 \\
train (seat) Z & 32.07 & 24.64 & 32.15 & 23.40 \\
\hline
\end{tabular}

\subsection{Comments}

From Table 4 it can be seen that if we want to achieve SNR higher than $23 \mathrm{~dB}$ for all considered vibration signals, we need to use a PCM system with 256 quantization levels, i.e. with a bit rate of $8 \mathrm{bps}$. For some signals that have narrow variance range close to the referent variance, values of SNR much larger than $23 \mathrm{~dB}$ are achieved.

Using the APCM system, the SNR of $23 \mathrm{~dB}$ for all observed signals can be achieved with the bit rate of $5 \mathrm{bps}$. The APCM system provides very close SNR values for all signals.

With the DPCM system, we need the bit rate of 4 bps to achieve SNR greater than 23 $\mathrm{dB}$ for all signals. Here we also can see large differences in SNR for different signals. Some signals with a narrow variance range and a very large correlation coefficient can achieve significantly high SNR values.

Using the ADPCM system, we can achieve SNR greater than $23 \mathrm{~dB}$ for all signals with the bit rate of $2 \mathrm{bps}$. In this case, there are also differences in the SNR between different signals due to the difference in the correlation coefficient.

We can see that the PCM system requires a much higher bit rate than other ADC systems. APCM system provides almost double reduction of the bit-rate, the DPCM system decreases the bit-rate for 2 times and ADPCM system provides the bit-rate decreasing of 4 times. This reduction in the bit- rate is particularly important in wireless 
measurement systems, due to limited bandwidth and lack of energy. If some other value for $\mathrm{SNR}_{\text {min }}$ is selected, some other values of the bit-rate would be obtained, but the ratio of the bit-rates for different ADC systems would be similar.

It should be noted that there are differences in the complexity of the considered ADC systems. The PCM system is the simplest, APCM and DPCM have greater complexity while the ADPCM system has the greatest complexity. Therefore, ADC systems that enable the bit-rate reduction are more complex. This means that a compromise must be made between the bit-rate reduction and complexity. This paper provides a detailed overview of these 4 ADC systems in terms of structure, design and performance, and provides engineers with the opportunity to choose the ADC system that is best suited for their specific application, taking into account both the performances (SNR and the bitrate) and complexity.

\section{CONCLUSION}

This paper presents the design and analysis of performances of four ADC systems (PCM, APCM, DPCM and ADPCM) for digitalization of vibration signals. Those systems have been known in literature and have been applied for some other types of signals (primarily for the voice signal), but in this paper those ADC models were optimized and designed specifically for vibration signals. To this aim, a detailed analysis of a larger number of vibration signals was done firstly, obtaining their main characteristics (variance range, range of correlation coefficient, range of variance of consecutive samples differences). It was shown that the variance of vibration signals can occupy a very wide range of about $30 \mathrm{~dB}$ while the variance of differences of consecutive samples can occupy the range of width of about $20 \mathrm{~dB}$. Also, it was shown that the vibration signals are highly correlated. Furthermore, since each vibration signal was quite long in time (several hundred thousand samples), it was found that characteristics of vibration signals can significantly vary during the time. Based on this analysis, parameters of four ADC systems, optimal for vibration signals, were determined. ADC systems are designed for the Gaussian distribution, since statistical characteristics of vibration signals and many other measurement signals are very well modeled by this type of distribution. The DPCM system was considered to exploit the high correlation of the vibration signals, while adaptive solutions were considered to cope with variations of signals' characteristics during the time (the APCM system is adaptive to signal variance while the ADPCM system is adaptive both on variance and correlation). Finally, an experiment was performed in which the designed ADC models were applied for the digitalization of 20 considered vibration signals. It was shown that SNR higher than $23 \mathrm{~dB}$ for all considered vibration signals can be achieved with the bit-rate of 8 bps with the PCM system, 5bps with the APCM system, 4 bps with the DPCM system and 2 bps with the ADPCM system. We can see that APCM, DPCM and ADPCM systems allow significant bit-rate reduction compared to the PCM system, but with the increasing of complexity, hence the compromise between the bit-rate reduction and complexity had to be done. The bit-rate reduction is particularly important from the aspect of application in wireless measurement systems for vibration measurement, due to the problem of lack of bandwidth and energy. 


\section{REFERENCES}

[1] M. Senldge, T. R. Llcht, Piezoelectric Acccelerometers and Vibration Preamplifiers - Theory and Application Handbook. Briiel\&Kiar, 1987.

[2] R. B. Randall, Vibration-based condition monitoring: industrial, aerospace and automotive applications. John Wiley \& Sons Ltd, 2011.

[3] E. Jantunen, "A summary of methods applied to tool condition monitoring in drilling," International Journal of Machine Tools \& Manufacture, vol. 42, pp. 997-1010, 2002.

[4] Mobius Institute, Vibration Analysis Definitions. [Online]. Available: http://www.mobiusinstitute. com/site2/item.asp?LinkID=2001

[5] F. Salvadori, M. de Campos, P. S. Sausen, R. F. de Camargo, C. Gehrke, C. Rech, M. A. Spohn, A. C. Oliveira, "Monitoring in Industrial Systems Using Wireless Sensor Network With Dynamic Power Management," IEEE Transactions on Instrumentation and Measurement, vol. 58, no. 9, pp. 3104 - 3111, 2009.

[6] B. Lu, L. Wu, T. G. Habetler, R. G. Harley, J. A. Gutiérrez, "On the Application of Wireless Sensor Networks in Condition Monitoring and Energy Usage Evaluation for Electric Machines," in Proceedings of 31st Annual Conference of IEEE Industrial Electronics Society, IECON 2005, Raleigh, NC, USA, 2005.

[7] X. Xue, V. Sundararajan, W. P. Brithinee, "The Application of Wireless Sensor Networks for Condition Monitoring in Three-phase Induction Motors," in Proceedings of IMECE 2006 ASME International Mechanical Engineering Congress and Exposition, Chicago, Illinois USA, 2006.

[8] N.S. Jayant, P. Noll, Digital Coding of Waveforms. Prentice-Hall, New Jersey, 1984.

[9] G. Petković, Z. Perić, V. Despotović, "Switched Uniform Scalar Quantization Adapted to Mean and Variance for Speech Coding," Facta Universitatis, Series: Automatic Control and Robotics, vol. 16, no. 3, pp. 263-274, 2017.

[10] B. Denić, Z. Perić, V. Despotović, "Forward Adaptive Speech Coding with Low Bit Rates and Variable Word Length," Facta Universitatis, Series: Automatic Control and Robotics, vol 15, no. 2, pp. 125-136, 2016.

[11] M. Dinčić, D. Denić, Z. Perić, D. Gleich, P. Planinšič, V. Dimčev, "A method for decreasing the number of bits for digital representation of measurement signals," ETAI 2015, Ohrid, Makedonija, 2015.

[12] I. Neri, et al., "A real vibration database for kinetic energy harvesting application," Journal of Intelligent Material Systems and Structures, vol. 23, no. 18, pp. 2095-2101, 2012. 